\title{
An Improved Particle Swarm Optimizer Based on Thermodynamical Model
}

\author{
NIE Xin and LI Yuan-xiang
}

\begin{abstract}
After the brief review of the basic principles and characteristics of Particle Swarm Optimization (PSO), a new particle swarm optimization, based on the simple evolutionary equations and the steep thermodynamical selection rule, are proposed to alleviate the premature convergence. The algorithm based on thermodynamical model, in which the selection rule simulates the competitive mechanism between energy and entropy in annealing to modify the exploitation and the exploration adaptively, can produce less off-particles in different free-energy scale not only to prevent the swarm from clustering and reduce the computational cost, but also to vary the diversity of the swarm and contribute to a global optimum output in the swarm. Relative experiments have been done; the results show the improved PSO performs very well on benchmark problems, and outperforms the other related PSO in search ability and stability.
\end{abstract}

Index Terms-Thermodynamical model, particle swarm optimization, entropy, swarm diversity

\section{INTRODUCTION}

The Particle Swarm Optimization (PSO) is an evolutionary computation technique developed by Kennedy and Eberhart $^{[1]}$. The underlying motivation for the development of PSO algorithm was social behavior of animals such as bird flocking, fish schooling, and swarm theory. A population of random solutions initialized in the beginning of the algorithm, called particles. Each particle is treated as a point in a D dimensional search space. During a search process, each particle has a tendency to fly towards better search areas with a velocity, which is dynamically adjusted according to its own and its companion's historical behavior.

According to standard PSO model ${ }^{[2]}$, the flying of a particle is described by its velocity and position, and the update equations is defined as the following:

$$
\begin{gathered}
v_{i d}(t+1)=w v_{i d}(t)+c_{1} r_{1}\left(p_{i d}(t)-x_{i d}(t)\right)+c_{2} r_{2}\left(p_{g d}(t)-x_{i d}(t)\right) \\
x_{i d}(t+1)=x_{i d}(t)+v_{i d}(t+1)
\end{gathered}
$$

Suppose that the particles fly through a D dimensional space, $\mathrm{X}_{\mathrm{i}}$ and $\mathrm{V}_{\mathrm{i}}$ represent the position vector and velocity

Manuscript received November 28, 2010; revised March 2, 2011. This work was supported in part by the National Natural Science Foundation No. 61070009

NIE Xin is currently a Ph.D candidate of Computer Software and Theory for Research in Computational Intelligence and Applications, Wuhan University, P.R. China. (e-mail: nix83@163.com).

LI Yuan-xiang is currently a professor of Computer Software and Theory for Research in Computational Intelligence and Applications, Wuhan University, P.R. China. vector of the $i$-th individual respectively, while $\mathrm{P}_{\mathrm{i}}$ and $\mathrm{P}_{\mathrm{g}}$ represent the $i$-th individual's best position ever found and the group's best position; the inertia weight $\omega$ is a scaling factor controlling the influence of the old velocity; $C_{1}$ and $C_{2}$ are positive constants known as "cognitive" and "social" coefficients which determine the weight of $\mathrm{P}_{\mathrm{i}}$ and $\mathrm{P}_{\mathrm{g}}$ respectively; $r_{1}$ and $r_{2}$ are two random numbers sampled from a uniform distribution in the range $[0,1]$ separated.

As a stochastic algorithm, PSO own some attractive features, but there are still some demerits puzzling researchers especially in some multidimensional complex space. In order to alleviate the premature phenomenon, a feasible idea is to enhance the whole performance of algorithms by means of maintaining an appropriate swarm-diversity during a search. Some techniques have been proposed following the idea. Particle swarm optimization with simulated annealing (SAPAO) $)^{[3]}$ utilized the thought concerning annealing process operation to enhance the local search ability around the optimum ${ }^{[4]}$.A dissipative particle swarm optimization (DPSO), which introduces negative entropy to construct an opening dissipative system, is developed according to the self-organization of dissipative structure to improve the swarm-diversity ${ }^{[5]}$. Self-organizing hierarchical particle swarm optimization (HPSO) introduced the concept of "mutation" to enhance the global search capability of the particles by providing additional diversity; only the "social" part and the "cognitive" part of the particle swarm strategy are considered to estimate the new velocity of each particle and particles are reinitialized whenever they are stagnated in the search space. Although these methods improve the algorithm performance to a certain extent, few can get over the problems about premature convergence and the computation cost truly.

In this paper, a steep thermodynamical particle swarm optimization (STPSO) incorporating with a new selection rule and the simplified evolutionary equation in order to get rid of these defects is presented. The new selection rule, which is based on the minimal free energy principle of statistical mechanics, simulates the competitive mechanism between energy and entropy in annealing to control the swarm-diversity. The remaining of the paper is organized as follows. Section 2 provides some relative improvement on PSO. Section 3 describes the STPSO in details. Section 4 presents the relative experimental results. Finally, Section 5 concludes with some remarks.

\section{The SimplificAtion OF PARTICle SWARM OPTIMIZATION}

Through carefully analyzing the PSO biological model, 
and evolution iterative equation (1) (2), It is easily found that particle velocity concept is not a prerequisite for the evolution process. Literature ${ }^{[6]}$ has proved that the PSO evolution procedure is independent of the particle velocity, consequently, it is appropriate to discard the particle velocity and the evolutionary process is only controlled by the variables of the particles position. the simplified optimal equation without the velocity is written as:

$$
x_{i d}(t+1)=w x_{i d}(t)+c_{1} r_{1}\left(p_{i d}(t)-x_{i d}(t)\right)+c_{2} r_{2}\left(p_{g d}(t)-x_{i d}(t)\right)
$$

The right side of equation (3) consists of three parts: the first part is the "history" part, which represents influence of previous position on current one while the extent of influence is controlled by the inertia weight $\omega$; the second part is the "cognition" part, which represents the private thinking of the particle itself; the third part is the "social" part, which represents the collaboration among the particles.

\section{STEEP THERMODYNAMICAL PARTICLE SWARM OPTIMIZATION}

Combined with the above simplified iteration equation (3), STPSO introduces a steep thermodynamical selection strategy which simulates the competitive mechanism between energy and entropy in annealing to modify the exploitation and the exploration adaptively.

\section{A. The Principle of Minimum Free Energy}

As we known, y is followed through all transition of the system at each temperature. It can be described as followed:

For a closed system that exchange heat with ambience to preserve the temperature unchanged, the states always diversify spontaneously into a lower total free energy and the system achieves equilibrium when its free energy seeks a minimum. In thermodynamics, the free energy formula is defined as ${ }^{[7]}$ :

$$
F=E-H T
$$

where $E$ is the energy of the system, $H$ is its entropy, and $T$ is the temperature which determines the relative weights between energy and entropy in the competition. The concept about entropy is proposed to quantificationally measure the energy dispersal of particles in the system.

\section{B. Measurement of Swarm Diversity}

It is a crucial issue how to measure population diversity while introducing the tournament mechanism into STPSO. In STPSO, we give some definitions of how to calculate the free energy of each particle and propose a level-based entropy while the extension field is graded on the basis of energy.

Suppose that $\mathrm{S}$ represents the search space and $\mathrm{f}(\mathrm{x})$ an objective function, we take $\mathrm{N}$ particles to compose the swarm population which remarks as $P_{t}=\left\{X_{1}, X_{2}, \ldots \ldots, X_{N}\right\}$ at iterative step $\mathrm{t}$, and the number of sub-population size is $\mathrm{M}^{[8]}$.

Definition 1. While each particle $X_{r} \in S$, the raw energy of individual is defined as

$$
S E\left(X_{r}\right)=\left\{\begin{array}{cc}
f\left(X_{r}\right) ; & \text { for the minimum problem } \\
-f\left(X_{r}\right) ; & \text { for the maximum problem }
\end{array}\right.
$$

Definition 2. At the iteration $t$, the extension field $W t=\left[l_{t}, u_{t}\right]$ is a sheer energy range of all particles which generate from the evolution beginning to the $\mathbf{t}$-th iteration in the search process, and assumed $u_{t}$ and $l_{t}$ are respectively an upper bound and a lower bound of the particle energy. The extension field is divided into $\mathrm{K}$ grids, they can be marked as $\beta_{1}, \beta_{2}, \ldots . ., \beta_{K}$, which satisfy,

$$
\begin{gathered}
\beta_{t}^{i} \cap \beta_{t}^{j}=\varnothing \\
\cup \beta_{t}^{i}=W_{t},(0 \leq i \leq K-1) \\
\beta_{t}^{i}=\left(\frac{a^{i-1}-1}{a^{K-1}-1} *\left(u_{t}-l_{t}\right)+l_{t}, \frac{a^{i}-1}{a^{K-1}-1} *\left(u_{t}-l_{t}\right)+l_{t}\right] \cap\left[l_{t}, u_{t}\right] \\
\text { herein } a>1, i \neq j, 0 \leq i, j \leq K-1, K \geq 2 .
\end{gathered}
$$

In these formula, "a" denotes proportional factorial constant. For every particle satisfies that $S E\left(X_{r}\right) \in \beta_{t}^{i}$, then we shall say that $X_{r}$ is at level $i$ of the iterative step $t$.

Definition 3. For arbitrary $X_{r} \in P_{t}$, then the relative energy of the particle is defined as

$$
R E\left(W_{t}, X_{r}\right)=\frac{a^{i}-1}{a^{K-1}-1}, \text { while } S E\left(X_{r}\right) \in \beta_{t}^{i} \text {. }
$$

Definition 4. Assume the number $n_{i}$ should be calculated as the particles which are at level i of the range $W_{t}$. Therefore, the level-based entropy $\mathrm{H}$ is defined as:

$$
H\left(W_{t}, P_{t}\right)=\sum_{i=0}^{K-1} \frac{n_{i}}{N} \log _{k} \frac{n_{i}}{N}
$$

The concept of level-based entropy, independent of the particle coding mode, is introduced to measure dispersal of the particles fitness at lower computational cost. We can prove that the entropy is calculated as 0 when all particles of swarm are located at the same level, or as the big number 1 when the particle number of each level is the same, namely, $n_{1}=n_{2}=\ldots=n_{K-1}$.

Definition 5. At the given iteration $\mathrm{t}$, it is known that the swarm $P_{t}$ and the extension field ${ }{ }_{t}$, the population energy $\mathrm{E}$ and the free energy $\mathrm{F}$ at temperature $\mathrm{T}$ are defined as:

$$
\begin{gathered}
E\left(W_{t}, P_{t}\right)=\frac{1}{N} \sum_{X_{r} \in P_{t}} \operatorname{RE}\left(W_{t}, X_{r}\right) \\
F\left(W_{t}, T, P_{t}\right)=E\left(W_{t}, P_{t}\right)-T H\left(W_{t}, P_{t}\right) .
\end{gathered}
$$

Definition 6. For every $X_{r} \in P_{t}$ at level i( $\left.\beta^{i}{ }_{t} \subset W_{t}\right), \mathrm{n}_{\mathrm{i}}$ is the number of particles located in the scale $\beta^{i}$, then the free energy component at temperature $\mathrm{T}$ is defined as: 


$$
F_{c}\left(W_{t}, T, P_{t}\right)=R E\left(W_{t}, P_{t}\right)+T \log _{K}\left(\frac{n_{i}}{N}\right)
$$

The free energy component $F_{c}$ for each particle obtains the minimum; as a result, the minimization of the swarm free energy $F$ descends most deeply. Consequently, in the selection rule, the free energy component is used to evaluate particles not according to the fitness value.

\section{Outline of STPSO}

Based on the analysis above, the pseudo code of SOPSO can be described as follows. In the procedure, the flying times of particles is controlled by the parameter (Temperature_changeCritical) at each temperature.

\section{Procedure STPSO}

(1) Initialize a particle swarm system, $t=0$ :

(a) Configure some parameters such as proportional factorial, the level total number, and the initial temperature etc;

(b) Randomly generate an initial population $P_{0}=\left\{X_{1}, X_{2}, \ldots \ldots, X_{N}\right\}$ and evaluate them;

(c) Save each particle's best extremun and the group's best extremun in the swarm $P_{0}$;

(d) Determine the energy bounds $u_{0}$ and $l_{0}$ for the initial extension field;

(2) Execute iteration

\section{Repeat}

(a) parameter_k $=0$

Repeat

(b) Configure $L_{k}=0$;

(c) Randomly choose parent particles from the current swarm to generate $\mathrm{M}$ offsprings by the simplified iteration equations and evaluate them; (d) Produce new extension field ${ }^{W_{t+1}}$ for the iteration $\mathrm{t}+1$;

(e) Calculate the free energy component $F_{c}$ for each particles including $P_{t}$ and $O_{t}$. If the offspring free energy component is less than its parent particle one, then the offspring replaces the parent particle to form the next generation $P_{t+1}$;

(f) Update each particle's best extremun and the group's best extremun of the current swarm ${ }^{{ }^{t+1}}$;

(g) $\mathrm{t}=\mathrm{t}+1, L_{k}=L_{k}+1$;

until $\left\{L_{k}<\right.$ Temperature_changeCritical \}

(a) parameter_k $=$ parameter_k $\mathrm{k}+1$;

(b) $\mathrm{T}=\mathrm{T} /(1+\overline{\mathrm{k}})$;

until\{ Termination_test $\left({ }^{P_{t}}\right)==$ False $\}$

\section{EXPERIMENTAL StUDIES}

\section{A. The Benchmark Functions}

In this section, we apply the standard particle swarm optimization (PSO) ${ }^{[9]}$, the hybrid algorithm based on PSO and simulated annealing (SAPSO) and STPSO to solve some typical numerical function minimization problems in Table 1.The following benchmark functions have been used to verify the performances of the three kinds of algorithms adopting different selection strategies in the comparative experiments. They are multimodal functions with many local optima and the basic information in experiments is listed in Table.1.

TABLE 1 THE BENCHMARK FUNCTIONS

\begin{tabular}{|c|c|c|c|c|}
\hline Name & Formula & $\begin{array}{c}\text { Dim } \\
n\end{array}$ & $\begin{array}{c}\text { Range } \\
\text { [Xmin, Xmax] }\end{array}$ & Optimal \\
\hline Griewank & $f_{1}(x)=\frac{1}{4000} \sum_{i=1}^{n}\left(x_{i}\right)^{2}-\prod_{i=1}^{n} \cos \left(\frac{x_{i}}{\sqrt{i}}\right)+1$ & 30 & {$[-100,100]^{30}$} & 0 \\
\hline Rastrigin & $f_{2}(x)=\sum_{i=1}^{n-1}\left(x_{i}^{2}-10 \cos \left(2 \pi x_{i}\right)+10\right)$ & 30 & {$[-600,600]^{30}$} & 0 \\
\hline Shaffer & $f_{3}(x)=0.5+\frac{\left(\sin \sqrt{x^{2}+y^{2}}\right)^{2}-0.5}{\left(1.0+0.001\left(x^{2}+y^{2}\right)\right)^{2}}$ & 2 & {$[-100,100]^{30}$} & 0 \\
\hline
\end{tabular}

\section{B. Experiments and Results}

In the following experiments, all optimization algorithms utilized the uniform termination condition for reasonable comparison with diverse selection strategies of algorithms. The common parameters are set as follows: population size $\mathrm{N}=80, \mathrm{C} 1=\mathrm{C} 2=2$, and $V_{\max }=20$, the inertia weight $\omega_{\text {is a }}$ random number separated in the uniform distribution $[0,1]$, the termination criterion is satisfied when the executions run up to the maximal iterative steps 10000 . Some additional parameters are fine tuned in each test algorithm: the initial temperature $\mathrm{T}=100$ and annealing rate $\alpha=0.9$ for the SAPSO; the offspring population for STPSO has the size $\mathrm{M}=$ 4 , and level number $\mathrm{k}=10$, chain length $L_{k}=2000$. The chaotic factors $c^{v_{v}}$ and $c_{l}$ are set as 0.002 for one parameter and as 0 for another parameter to test different status, respectively.

Under these settings, we executed the programs 50 times consecutively. The statistic results are showed in the follows.Table3 provides the statistic results of 50 trials for each algorithm. The results of Table 1 demonstrate clearly 
the stability of STPSO. Its successful convergence rate is much high than that of the other two algorithms. Moreover, the quality of its solutions is averagely superior to that of the others due to its stability and it even can be seen that the worst fitness value for STPSO outperforms the others by several magnitudes on all benchmark functions - it is clearly capable of escaping local optima.

TABLE 2 Statistic Results on the Benchmark FunCtions For ThreE Algorithms

\begin{tabular}{cccccc}
\hline Algorithm & Benchmark Function & Convergence Rate & Mean Result & Best Result & Worst Result \\
\hline \hline PSO & $f_{1}$ & $13 / 50$ & $1.677229 \mathrm{e}-002$ & $0.000000 \mathrm{e}+000$ & $1.028944 \mathrm{e}-001$ \\
DPSO & $f_{1}$ & $6 / 50$ & $4.812129 \mathrm{e}-002$ & $0.000000 \mathrm{e}+000$ & $1.683775 \mathrm{e}-001$ \\
STPSO & $f_{1}$ & $48 / 50$ & $4.440892 \mathrm{e}-018$ & $0.000000 \mathrm{e}+000$ & $1.110223 \mathrm{e}-016$ \\
\hline PSO & $f_{2}$ & $0 / 50$ & $1.343209 \mathrm{e}+001$ & $1.989918 \mathrm{e}+000$ & $2.984875 \mathrm{e}+001$ \\
DPSO & $f_{2}$ & $45 / 50$ & $9.949591 \mathrm{e}-002$ & $0.000000 \mathrm{e}+000$ & $9.949591 \mathrm{e}-001$ \\
STPSO & $f_{2}$ & $48 / 50$ & $1.065814 \mathrm{e}-016$ & $0.000000 \mathrm{e}+000$ & $3.552714 \mathrm{e}-015$ \\
\hline PSO & $f_{3}$ & $0 / 50$ & $9.646106 \mathrm{e}+000$ & $5.395604 \mathrm{e}-002$ & $1.479383 \mathrm{e}+001$ \\
DPSO & $f_{3}$ & $50 / 50$ & $2.671562 \mathrm{e}-006$ & $2.671541 \mathrm{e}-006$ & $2.672180 \mathrm{e}-006$ \\
STPSO & $f_{3}$ & $50 / 50$ & $2.671541 \mathrm{e}-006$ & $2.671541 \mathrm{e}-006$ & $2.671541 \mathrm{e}-006$ \\
\hline
\end{tabular}

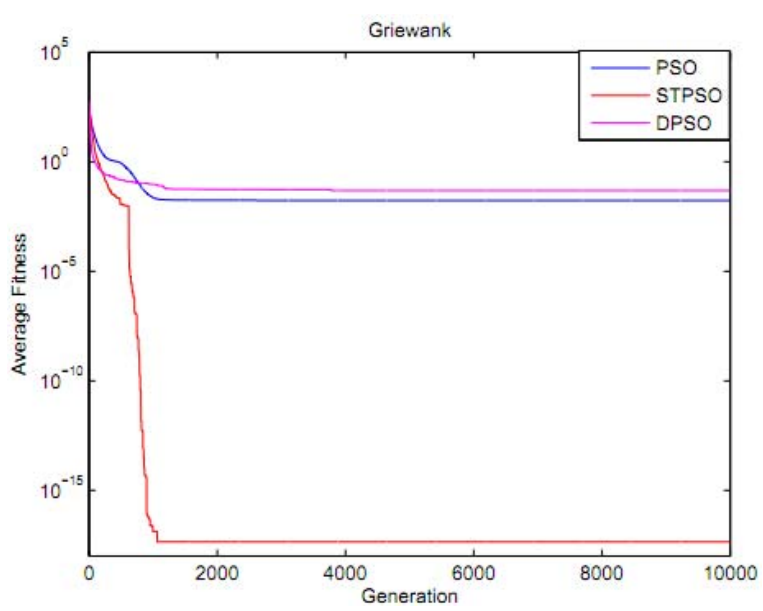

Fig. 1. Average fitness evolutionary curves of $f_{1}$

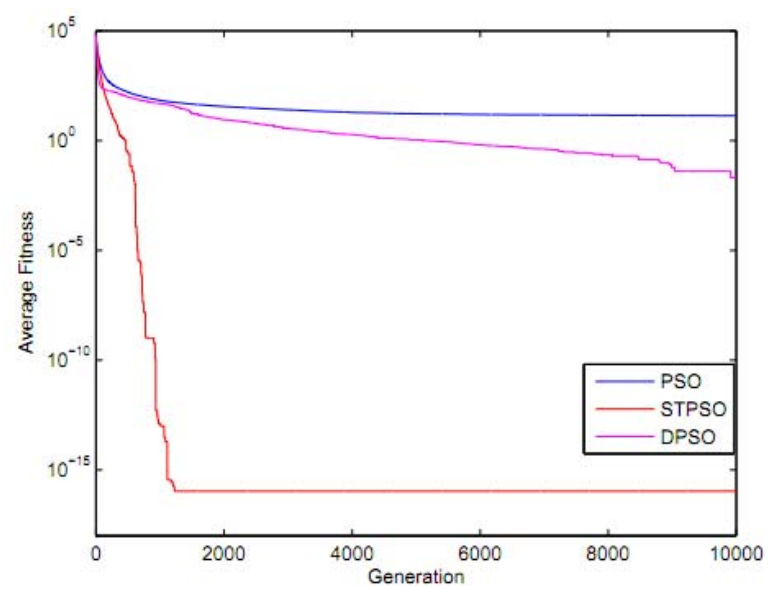

Fig. 2. Average fitness evolutionary curves of $f_{2}$

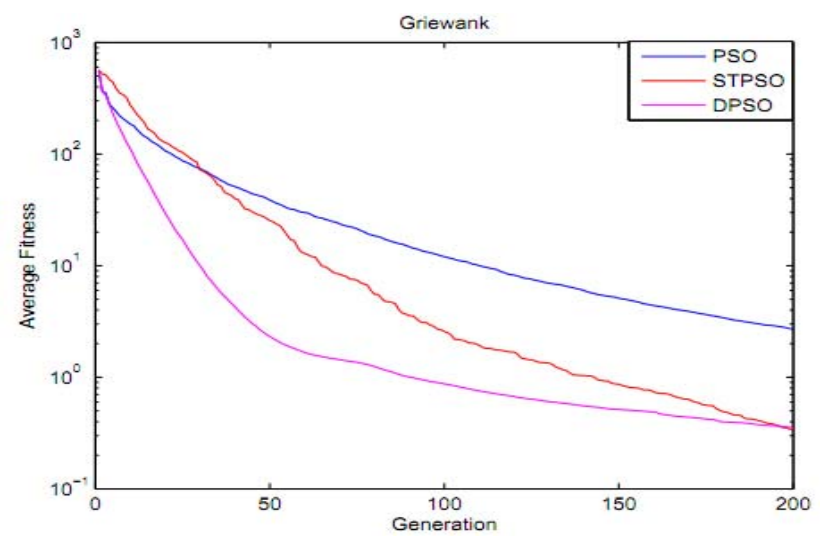

Fig. 3. Average fitness evolutionary curves at early stage of $f_{1}$

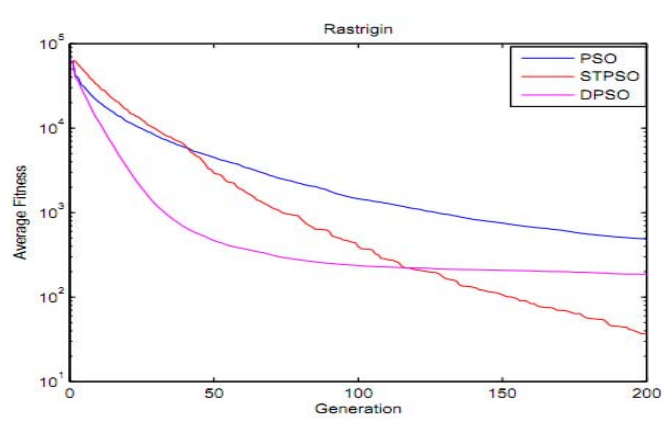

Fig. 4. Average fitness evolutionary curves at early stage of $f_{2}$

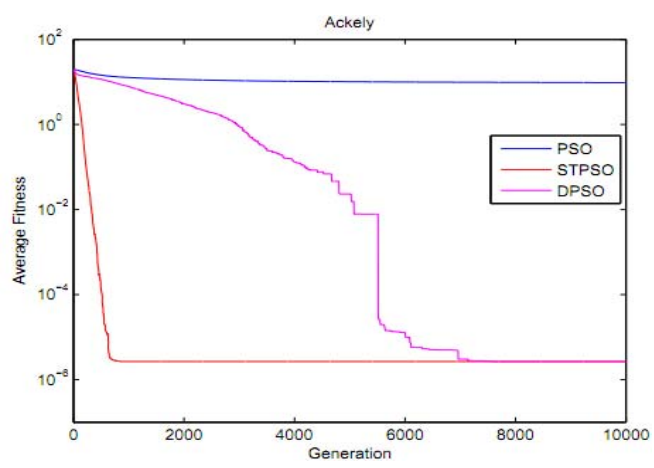

Fig. 5. Average fitness evolutionary curves of $\mathrm{f}_{3}$

Figure 1, 2 show their average convergence curves in 50 trials of the best individual of each generation, and Figure 3,4 show the best fitness average convergence curves of each generation at the initial evolutionary stages. The STPSO kept on optimizing towards a better fitness, whereas the other algorithms stagnated and flattened out with no further improvement. Some analysis can be made on the basis of the experiments: Regarding the Griewank function (figure 3), the convergence speeds of STPSO are slower than DPSO at the early 200 iterations. One of the main reason is that the number of generated new particles is much less in the STPSO evolutionary process, namely, the new particles number of the other algorithms are twenty times much more than the one of STPSO at each generation. However, observing on figure 1, after 1800 Iterations the PSO and DPSO optimizations obtained no further improvements, whereas the STPSO was still improving notably and converged to a significantly better end-result. The analogous situation can be observed from the figure 2,4 concerning Rastrigin test function. The average convergence curve (figure 5) of STPSO regarding the Ackley function has lower running timesteps. The 
simplified evolutionary equation and the steep thermodynamical selection strategy are the basilica reasons. The former economized the computational time and the selection rules insure the swarm diversity against getting trapped at the local optima and against the premature convergence.

\section{CONCLUSION}

This paper has developed a new particle swarm optimization based on thermodynamical model, which is based on the simplified updating formula and the steep thermodynamical selection strategy, and evaluated its performance on a number of benchmark problems. The selection strategy simulates the competitive mechanism between energy and entropy in annealing to modify the exploitation and exploration adaptively. The relative experimental results show that the improved algorithm outperforms PSO and DPSO for the complex optimization problems, especially for the multimodal functions in a high dimension space. Through the new selection rules, it is not only susceptible to escape from the local optima and convenge to the global optimum at the very little cost of the early stage, but also improves the stability and the computational efficiency greatly.

\section{ACKNOWLEDGMENT}

We would like to thank State Key Laboratory of Software Engineering, Wuhan University for providing us research opportunities and their wholehearted support for such activities. Finally, our acknowledgement cannot end without thanking to the authors whose research papers helped us in making this research.

\section{REFERENCES}

[1] Kennedy, J. and Eberhart, R.C., "Particle swarm opti-mization", IEEE International Conference on Neural Networks, IEEE Press, (1995),pp.1942-1948

[2] Xie XF, Zhang WJ, Yang ZL, "A dissipative particle swarm optimization",IEEE International Conference on Evolutionary Computation, IEEE Prss, Honolulu, (2002),pp.1456-1461

[3] Shi, Y. and Eberhart, R.C, "A modified particle swarm optimizer", IEEE International Conference on Evolutionary Computation, IEEE Press, Piscataway, (1998),pp.69-73

[4] Jing Jie, Jianchao Zeng, Chongzhao Han, "Self-Organization Particle Swarm Optimization Based on Information Feedback", ICNC, Springer Berlin, 4221(2006),pp. 913-922

[5] Gao Shang, Yang Jingyu, Wu Xiaojun, "Particle Swarm Optimization based on the Ideal of Simulated An-nealing Algorithm", Computer Applications and Software,1(2005),pp.104

[6] Hu Wang, Li Zhishu, "A Simpler and More Effective Particle Swarm Optimization Algorithm",Journal of Software, (4)18(2007),pp.861.868.

[7] Kirkpatrick, S., Gelatt, C.D., Vecchi, M.P., “ Optimization by simulated annealing", Science, 220(1983),pp.671-680

[8] Li, T. Wei, C. and Pei, W, "PSO with sharing for multimodal function optimization", IEEE International Conference on Neural Networks and Signal Processing, IEEE Press, 1(2003),pp.450C453.

[9] C.-H. Im, H.-K. Kim, and H.-K. Jung, "A novel algorithm for multimodal function optimization based on evolution strategy", IEEE Transaction on Magnetics, IEEE Press, 3(2004), pp.1224C1227, .

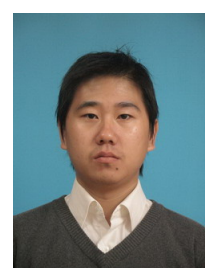

NIE Xin received the B.S. degree in computer science and the M.S. degree in software engineering from Wuhan University, Wuhan, P.R. China, in 2003, and 2006 respectively. He is currently a Ph.D candidate of Computer Software and Theory for Research in Computational Intelligence and Applications, Wuhan University, China. His main research interests include the evolutionary computations, reconfigurable hardware, evolvable hardware, bio-inspired computing and applying this to complex real-world applications. 